\title{
There is a unique crossing-minimal rectilinear drawing of $K_{18}$
}

\author{
Oswin Aichholzer \\ Institute for Software Technology, University of Technology, Graz, Austria \\ Bernardo M. Ábrego \\ Silvia Fernández-Merchant \\ Departament of Mathematics, California State University at Northridge, CA, \\ United States \\ Jesús Leaños \\ Unidad Académica de Matemáticas, Universidad Autónoma de Zacatecas, México \\ Gelasio Salazar \\ Instituto de Física, Universidad Autónoma de San Luis Potosí, México
}

\begin{abstract}
We show that, up to isomorphism, there is a unique crossing-minimal rectilinear drawing of $K_{18}$. As a consequence we settle, in the negative, the following question from Aichholzer and Krasser: does there always exist an crossing-minimal drawing of $K_{n}$ that contains a crossing-minimal drawing of $K_{n-1}$ ?
\end{abstract}

Keywords: Rectilinear crossing number, complete graphs, $k$-edges 


\section{Introduction}

The rectilinear crossing number $\overline{\mathrm{cr}}(G)$ of a graph $G$ is the minimum number of edge crossings in a rectilinear (or geometric) drawing of $G$ in the plane, i.e., a drawing of $G$ in the plane where the vertices are points in general position and the edges are straight segments. A drawing of $G$ with exactly $\overline{\mathrm{cr}}(G)$ crossings is crossing-minimal.

Determining the rectilinear crossing number $\overline{\mathrm{cr}}\left(K_{n}\right)$ of the complete graph $K_{n}$ is a well-known open problem in combinatorial geometry (see for instance [9]). In [7] Aichholzer et al. announced the exact determination of $\overline{\operatorname{cr}}\left(K_{n}\right)$ for $13 \leq n \leq 17$. In that paper also the following question was raised.

Question 1.1 Is it true that, for every integer $n \geq 4$, there exists an crossingminimal drawing of $K_{n}$ that contains an crossing-minimal drawing of $K_{n-1}$ ?

The exact value of $\overline{\mathrm{cr}}\left(K_{n}\right)$ is known for $n \leq 27$ and $n=30$ (see $[1,5,6,7,8]$ ). In particular, $\overline{\mathrm{cr}}\left(K_{18}\right)=1029$ was established in [6]. Crossing-minimal rectilinear drawings of $K_{n}$ for this range of values of $n$ can be found in [2] and [4].

Let $\theta$ denote the counterclockwise rotation of $2 \pi / 3$ around the origin, and let $W:=\{(-51,113),(6,834),(16,989),(18,644),(18,1068),(22,211)\}$. Then (see [2]) the 18-point set $W \cup \theta(W) \cup \theta^{2}(W)$ induces an crossing-minimal drawing of $K_{18}$.

Our main result is the following.

Theorem 1.2 Up to order type isomorphism, there is a unique 18-point set whose induced rectilinear drawing of $K_{18}$ has $\overline{\mathrm{cr}}\left(K_{18}\right)$ crossings.

Let $\mathcal{D}$ be the (unique, in view of Theorem 1.2) crossing-minimal geometric drawing of $K_{18}$. It is easily verified that every subdrawing of $\mathcal{D}$ with 17 points has more than $\overline{\mathrm{cr}}\left(K_{17}\right)=798$ crossings. This settles Question 1.1 in the negative.

In the next section, we introduce the necessary notation and additional concepts required for the proof of Theorem 1.2. In Section 3 we give a brief sketch of the proof of Theorem 1.2.

\section{$2 \quad k$-edges, $(\leq k)$-edges, and 3-decomposability}

Let $Q$ be a point set in the plane. If $p, q \in Q$, we denote by $p q$ the straight line segment with end points $p$ and $q$. We use $\ell(p q)$ to denote the directed line that spans $p$ and $q$, directed from $p$ towards $q$. Furthermore, $\ell(p q)^{+}$and $\ell(p q)^{-}$denote the halfplanes to the right and left, respectively, of $\ell(p q)$. 
Let $Q$ be an $n$-point set in the plane in general position, and let $0 \leq k \leq$ $n / 2-1$. A $k$-edge of $Q$ is a line that spans two points of $Q$, and leaves exactly $k$ points on one side. A $(\leq k)$-edge (respectively, a $(>k)$-edge) is an $i$-edge with $0 \leq i \leq k$ (respectively, $k<i \leq n / 2-1$ ). Let $E_{k}(Q), E_{\leq k}(Q)$, and $E_{>k}(Q)$ denote, respectively, the number of $k$-edges, $(\leq k)$-edges and $(>k)$-edges of $Q$. Note that $E_{\leq k}(Q)=\sum_{j=0}^{k} E_{j}(Q)$ and $E_{>k}(Q)=\left(\begin{array}{c}n \\ 2\end{array}\right)-E_{\leq k}(Q)$. The vector $v_{k}(Q):=\left(E_{0}(Q), E_{1}(Q), \ldots, E_{\lfloor n / 2\rfloor-1}(Q)\right)$ is the vector of $k$-edges of $Q$. The vector $v_{\leq k}(Q)$ of $(\leq k)$-edges of $Q$ is analogously defined. Finally, $E_{\leq k}(n)$ denotes the minimum of $E_{\leq k}(Q)$ taken over all $n$-point sets $Q$. The exact determination of $E_{\leq k}(n)$ is another open problem in combinatorial geometry (see $[1,3,5,6])$.

The number of crossings in a geometric drawing of $K_{n}$ and the number of $k$ - and $(\leq k)$-edges in its underlying $n$-point set $P$ are closely related by the following equality, independently proved in [3] and [10]:

$$
\overline{\operatorname{cr}}(P)=\sum_{k=0}^{\lfloor n / 2\rfloor-1}(n-2 k-3) E_{\leq k}(P)-\frac{3}{4}\left(\begin{array}{l}
n \\
3
\end{array}\right)+\left(1+(-1)^{n+1}\right) \frac{1}{8}\left(\begin{array}{l}
n \\
2
\end{array}\right) .
$$

Finally, we introduce a concept that captures a property shared by all known crossing-minimal geometric drawings of $K_{n}$, for $n$ a multiple of 3. A point set $P$ is 3-decomposable if it can be partitioned into three equal-size sets $A, B$ and $C$, such that (i) there exist a triangle $T$ enclosing the point set $P$; and (ii) the orthogonal projection of $P$ onto the three sides of $T$ shows $A$ between $B$ and $C$ on one side, $B$ between $C$ and $A$ on the second side, and $C$ between $A$ and $B$ on the third side. In this context, $\{A, B, C\}$ is a 3-decomposition of $P$.

\section{Uniqueness of crossing-minimal drawing of $K_{18}$}

Throughout this section, $\mathcal{D}$ is an crossing-minimal rectilinear drawing of $K_{18}$, and $P$ is its underlying 18-point set.

Our strategy is as follows. First we show that the crossing-minimality of $\mathcal{D}$ completely determines $v_{\leq k}(P)$. We then argue that the entries of $v_{\leq k}(P)$ imply that $P$ must be 3 -decomposable. This in turn allows us to classify certain (as it happens, many) types of $k$-edges that must occur in $P$. Finally, we find a set of restrictions on the remaining $k$-edges, and show that they uniquely determine the order type of $P$. 
Sketch of Proof of Theorem 1.2 Using (1) and $\overline{\mathrm{cr}}\left(K_{18}\right)=1029$, it is not difficult to prove the following.

Proposition $3.1 v_{\leq k}(P)=(3,9,18,30,45,63,87,120,153)$.

We start by labeling the points of $P$. Since $E_{0}(P)=3$, then the convex hull of $P$ consists of exactly 3 points, say $a_{6}, b_{6}$ and $c_{6}$. Now we rotate $\ell\left(a_{6} c_{6}\right)$ from $c_{6}$ to $b_{6}$ around $a_{6}$ and for $i=1,2, \ldots, 5$, we let $c_{6-i}$ be the $i$-th point found by such a rotation. Similarly, we rotate $\ell\left(a_{6} b_{6}\right)$ from $b_{6}$ to $c_{6}$, again around $a_{6}$, and for $i=1,2, \ldots, 5$ we let $b_{6-i}$ be the $i$-th point found by such a rotation. Let $C:=\left\{c_{1}, \ldots, c_{6}\right\}, B:=\left\{b_{1}, \ldots, b_{6}\right\}$ and $A:=P \backslash B \cup C$. Clearly, $\{A, B, C\}$ is a partition of $P$.

From the entries of $v_{\leq k}(P)$ it follows that the same partition of $P$ is obtained if, instead of rotating around $a_{6}$, we rotate around $b_{6}$ or $c_{6}$. Moreover, for $\{x, y, z\}=\{a, b, c\}$, the numbers in $v_{\leq k}(P)$ imply that the rotations of $\ell\left(y_{6} x_{6}\right)$ and $\ell\left(z_{6} x_{6}\right)$ around $y_{6}$ and $z_{6}$, respectively, produce the same labels for the $x$ 's points, and so this labeling is well-defined. Note that $\{A, B, C\}$ is a 3-decomposition of $P$. In this context we define, as in [2], two types of edges. Let $p, q \in P$. If $p, q \in A, p, q \in B$ or $p, q \in C$ then we call $p q$ monochromatic; otherwise, $p q$ is bichromatic. Let $E_{<k}^{\text {mono }}(P)$ and $E_{<k}^{b i}(P)$ be the number of monochromatic and bichromatic $(\leq k)$-edges of $P$, respectively. Note that $E_{k}(P)=E_{k}^{\text {mono }}(P)+E_{k}^{b i}(P)$.

For $x \in\{a, b, c\}$, let us denote the number of monochromatic $(>k)$-edges of type $x x$ by $E_{>k}^{x x}(P)$. Note that $E_{>k}^{m o n o}(P)=E_{>k}^{a a}(P)+E_{>k}^{b b}(P)+E_{>k}^{c c}(P)$.

Remark 3.2 Let $\{x, y, z\}=\{a, b, c\}$. Clearly, if we rotate $\ell\left(x_{6} y_{6}\right)$ around $x_{6}$ from $y_{6}$ to $z_{6}$, and $x_{\sigma(i)}$ is the $i$-th $x$ that is found by such a rotation, then $x_{6} x_{\sigma(i)}$ is a $j$-edge of $P$ for $j=\min \{5+i, 16-(5+i)\}$. Thus for $j=6,7$ there are exactly two $j$-edges of the type $x_{6} x$, and for $j=8$ there is exactly one 8-edge of the type $x_{6} x$.

The following is an immediate consequence of the 3-decomposability of $P$, Claim 1 in [2], and the fact that $E_{8}^{b i}(P)=108-\sum_{i=0}^{7} E_{i}^{b i}(P)$.

Proposition $3.3 E_{k}^{b i}(P)=3(k+1)$ for $k=0, \ldots, 5 ; E_{k}^{b i}(P)=18$ for $k=$ 6,7 ; and $E_{8}^{b i}(P)=9$.

Propositions 3.1 and 3.3 readily imply the following.

Corollary $3.4 E_{8}^{\text {mono }}(P)=24, E_{7}^{\text {mono }}(P)=15, E_{6}^{\text {mono }}(P)=6$, and $E_{k}^{\text {mono }}(P)=$ 0 for $k=0,1,2,3,4$, and 5 .

Claim 4 in [2] implies that $E_{8}^{x x}(P) \leq 8$ for each $x \in\{a, b, c\}$. Using this, together with Remark 3.2 and Proposition 3.4, we obtain the following. 
Proposition 3.5 Let $x \in\{a, b, c\}$. Then $E_{6}^{x x}(P)=2, E_{7}^{x x}(P)=5$, and $E_{8}^{x x}(P)=8$. Moreover, each 6-edge of type $x x$ involves $x_{6}$.

Using this last result and similar arguments, we obtain the following.

Proposition 3.6 Let $x \in\{a, b, c\}$. Then 1) $x_{6} x_{5}$ cannot be a 6-edge; 2) there are at least two 7 -edges of type $x x$ involving $x_{5}$ but not $x_{6}$; 3) $x_{6} x_{4}$ cannot be a 6-edge; 4) each element of $\left\{x_{3} x_{2}, x_{3} x_{1}, x_{2} x_{1}\right\}$ is an 8-edge; and 5) $x_{6} x_{2}$ and $x_{6} x_{1}$ are the two 6 -edges of type $x x$, i.e., $x_{3}, x_{4}$ and $x_{5}$ are contained in the triangle formed by $x_{6}, x_{2}$ and $x_{1}$.

We may assume that $x_{2} \in \ell\left(x_{6} x_{1}\right)^{-}$. Thus the triangle formed by $x_{6}, x_{2}$ and $x_{1}$ is as in Figure 1. Since $\ell\left(x_{3}, x_{1}\right)$ is an 8-edge, then exactly one element, say $w$, of $\left\{x_{4}, x_{5}, y_{1}, \ldots, y_{5}\right\}$ belongs to $\ell\left(x_{3}, x_{1}\right)^{-}$. A tedious but straightforward case analysis shows that $w$ must be $y_{1}$. Similarly, since $\ell\left(x_{2}, x_{1}\right)$ is an 8-edge, then there are exactly two $y$ 's in $\ell\left(x_{2}, x_{1}\right)^{-}$. Clearly, one of them is $y_{1}$. We can then deduce that the other $y$ must be $y_{2}$ and that $y_{2} \in \ell\left(y_{6} y_{1}\right)^{-}$. Then the points of $P$ with indices $1,2,3$ and 6 are as in Figure 1.

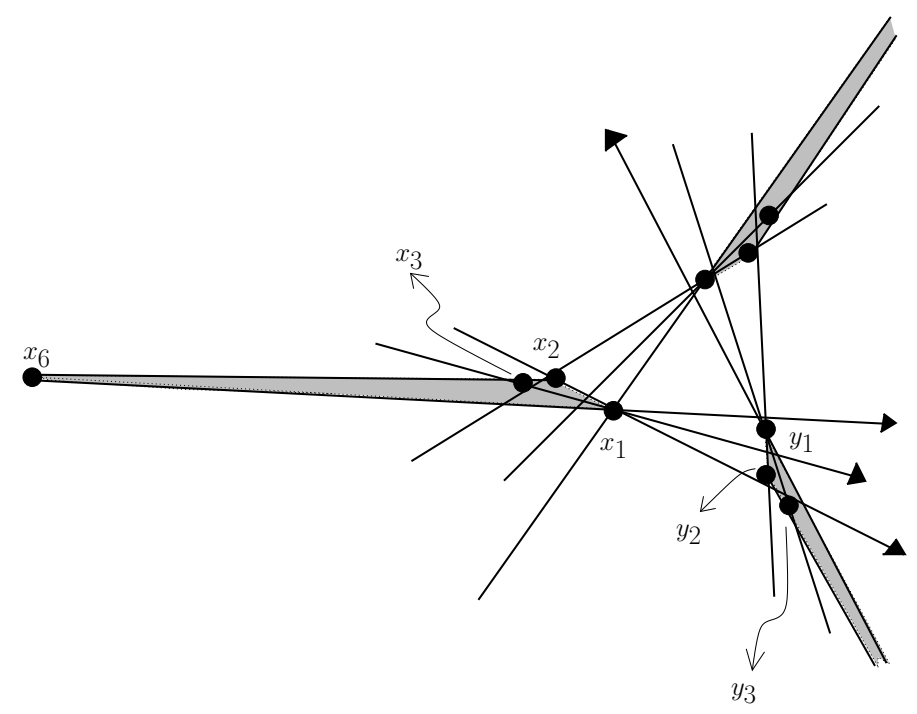

Fig. 1. The relative position of the points of $P$ with indices $1,2,3$ and 6 .

Using similar arguments one can prove the following.

Proposition 3.7 Let $x \in\{a, b, c\}$. Then 1) $x_{4} x_{2}$ cannot be an 8-edge; 2) $x_{6} x_{5}$ is an 8-edge; 3) $x_{3} \in \ell\left(x_{6} x_{5}\right)^{-}$and $x_{4} \in \ell\left(x_{6} x_{5}\right)^{+}$; 4) $x_{4} \in \ell\left(x_{2} x_{3}\right)^{-}$and $x_{5} \in \ell\left(x_{2} x_{3}\right)^{+}$; and 5) $x_{5} \in \ell\left(x_{1} x_{4}\right)^{-}$and $x_{3} \in \ell\left(x_{1} x_{4}\right)^{+}$. 
Finally, it is not difficult to see that the order type of $P$ is uniquely determined by the labeling of the points of $P$, the numbers in $v_{\leq k}(P)$, and the set of restrictions given by Propositions 3.5, 3.6, and 3.7.

\section{References}

[1] B. M. Ábrego, S. Fernández-Merchant, M. Cetina, J. Leaños and G. Salazar, On $\leq k$-edges, crossings, and halving lines of geometric drawings of $K_{n}$. arXiv:1102.5065v1 [math.CO].

[2] B. M. Ábrego, M. Cetina, S. Fernández-Merchant, J. Leaños and G. Salazar, 3 -symmetric and 3-decomposable geometric drawings of $K_{n}$. Discrete Applied Mathematics. 158 (2010) no. 12, 1240-1258.

[3] B. M. Ábrego and S. Fernández-Merchant, A lower bound for the rectilinear crossing number, Graphs and Comb. 21 (2005), no. 3, 293-300.

[4] O. Aichholzer, http://www.ist.tugraz.at/aichholzer/research /triangulations/crossing/.

[5] O. Aichholzer, J. García, D. Orden and P. Ramos, New lower bounds for the number of $(\leq k)$-edges and the rectilinear crossing number of $K_{n}$. Discrete Comput. Geom. 38 (2007), no. 1, 1-14.

[6] O. Aichholzer, J. García, D. Orden and P. Ramos, New results on lower bounds for the number of $(\leq k)$-facets, Electronic Notes in Discrete Mathematics 29 (2007), 189-193.

[7] O. Aichholzer and H. Krasser, Abstract order type extension and new results on the rectilinear crossing number. Comput. Geom. 36 (2007), no. 1, 2-15.

[8] M. Cetina, C. Hernández-Vélez, J. Leaños, and C. Villalobos, Point sets that minimize $(\leq k)$-edges, 3 -decomposable drawings, and the rectilinear crossing number of $K_{30}$, arXiv:1009.4736v1 [math.CO].

[9] R. K. Guy, A combinatorial problem, Nabla (Bulletin of the Malayan Mathematical Society) 7 (1960), 68-72.

[10] L. Lovász, K. Vesztergombi, U. Wagner and E. Welzl, Convex quadrilaterals and $k$-sets, Toward a Theory of Geometric Graphs, Contemp. Math., 342, Amer. Math. Soc. (2004), 139-148. 